\title{
Significance of Background Coloration in Endoscopic Detection of Early Esophageal Squamous Cell Carcinoma
}

\author{
H. Minami ${ }^{a} \quad$ H. Isomotob ${ }^{b} \quad$ H. Inoue ${ }^{d} \quad Y_{.}$Akazawa $^{a} \quad$ N. Yamaguchi ${ }^{b} \quad$ K. Ohnita ${ }^{a}$ \\ F. Takeshima ${ }^{\text {a }}$ T. Hayashic ${ }^{c}$ T. Nakayama ${ }^{c} \quad$ K. Nakao ${ }^{a}$ \\ Departments of a Gastroenterology and Hepatology, ${ }^{\mathrm{b}}$ Endoscopy and ${ }^{\mathrm{C} P a t h o l o g y, ~ N a g a s a k i ~ U n i v e r s i t y ~ H o s p i t a l, ~}$ \\ Nagasaki, and d Digestive Disease Center, Showa University Northern Yokohama Hospital, Yokohama, Japan
}

\section{Key Words}

Background coloration - Esophageal cancer - Squamous cell carcinoma - Narrow band imaging - Image-enhanced endoscopy $\cdot$ Magnifying endoscopy

\begin{abstract}
Endoscopic diagnostics of early squamous cell carcinoma (SCC) in the laryngo-esophageal region have dramatically improved together with development of less invasive endoscopic treatment. It is essential for gastrointestinal endoscopists to detect lesions when they are still endoscopically treatable, especially in this region since surgical approach can still be extremely invasive. Pioneers have found some notable fundamental alterations in early SCC and created several classifications. Inoue [Dig Endosc 2001;13(suppl): 40-41] proposed the intrapapillary capillary (IPCL) classification, which focused on the microvascular change of the mucosal surface. One of the significances of this classification is that it clearly distinguished the lesions that require further pathological evaluation by categorizing the diameter change of the IPCLs. On the other hand, Arima et al. [Esophagus 2005;2:191-197] advocated the alteration of microvessels as well as change of the vascular arrangement in the area. Most recently, the Japan Esophageal Society construct-
\end{abstract}

\section{KARGER}

E-Mail karger@karger.com www.karger.com/dig ed a new classification uniting these two exemplary classifications as the 'Japanese Classification of Magnifying Endoscopy for Early Squamous Cell Carcinoma'. This classification was intended to be simple and easily applicable in general clinical practice. Brownish color change between the IPCLs has reported to be one of the useful findings in distinguishing early SCC from benign changes such as inflammatory change and low-grade intraepithelial neoplasia. Nevertheless, the exact cause of this phenomenon remains unclear. We recently examined the association of color change with hemoglobin $(\mathrm{Hb})$ in cancer tissue, since NBI exclusively detects the wavelength of $\mathrm{Hb}$ in superficial vessels in the gastrointestinal tract. This review article also describes our examination of a distinct finding in esophageal cancer, namely, 'background coloration'.

(c) 2014 S. Karger AG, Basel

\section{Introduction}

In Japan, squamous cell carcinoma (SCC) accounts for more than $95 \%$ of esophageal cancer. Although the incidence of reflux esophagitis has increased, the frequency of esophageal adenocarcinoma has remained immutable, accounting for only about $4 \%$ of esophageal cancer [1]. The
(C) 2014 S. Karger AG, Basel

0012-2823/14/0891-0006\$39.50/0 
prognosis of esophageal SCC found in the late stages remains exceedingly poor because of the aggressive nature of the tumor [2]. The proportion of superficial carcinomas among esophageal malignancies in the last 10 years has been approximately 30-35\% (Japan Esophageal Society; comprehensive registry of esophageal cancer in Japan; http://esophagus.jp, accessed April 18, 2007). Although early detection results in better prognosis, it is challenging to detect flat lesions with slight color change. It is exceedingly difficult to find flat lesions, which can be treated using EMR (endoscopic mucosal resection) and ESD (endoscopic submucosal dissection) techniques, with slight color change using a conventional white-light image endoscope.

Chromoendoscopy using iodine has been one of the most reliable diagnostic tools in detecting early SCC of the esophagus $[3,4]$. It has been effectively utilized not only in detecting, but also determining the local extent of the tumor. However, iodine spraying often induces unpleasant side effects such as chest discomfort, cough and severe allergic reaction due to its high stimulation. Another issue to be mentioned is that detection of early lesions can be complicated by surrounding severely inflamed mucosa resulting from the patients' habit of drinking and smoking. Therefore, development of new technologies which allow us to obtain diagnostic information without harming patients is required. Recently, significant advancement has been made in the field of image-enhanced endoscopy such as magnifying endoscopy, narrow band imaging (NBI) and Fuji Intelligent Chromo Endoscopy. These newly developed modalities enable us to obtain detailed information of the gastrointestinal tract leading to more reliable evaluation that is consistent with pathological diagnosis.

Historically, several useful classifications, such as Inoue's intrapapillary capillary loop (IPCL) classification [5, 6] and Arima's microvascular patterns classification [7], have been proposed in Japan for diagnosis and evaluation of SCC. However, these two major classifications have been considered to have some difficulties because of their complexity. Thus, the Japan Esophageal Society decided to merge these two classifications into a simpler category that can easily be used in general clinical practice. They introduced the Japanese Classification of Magnifying Endoscopy for Early Squamous Cell Carcinoma as a comprehensive classification of superficial esophageal SCC [8]. Currently, new findings in addition to the vascular structure, such as brownish color change of the epithelia between each IPCL, have been introduced in the esophageal diagnostics. In this review article, we specifically focus on the endoscopic diagnosis of esophageal SCC.

Significance of BC in Endoscopic

Detection of Early Esophageal SCC

\section{Lugol Chromoendoscopy}

Ability to detect early squamous neoplasia of the esophagus can be improved considerably by iodine staining during endoscopic examination, especially for the high-risk population such as an over 50 -year-old male with a history of alcohol and tobacco abuse. Iodine-unstained areas usually indicate either inflammatory change, low-grade intraepithelial neoplasia (LGIN), high-grade intraepithelial neoplasia (HGIN), or invasive SCC. Mori et al. [9] analyzed the relationship between Lugol voiding areas and histological features. Their study demonstrated that the staining intensity reflected well the thickness of the glycogen-containing cell layer in the lesion. Furthermore, the sharpness of the margin after staining predicted whether the conversion from glycogen-containing mucosa to noncontaining cell layers was abrupt or gradually changed.

\section{Pink Color Sign/Metallic-Silver Sign}

Pink color sign (PCS) was first mentioned by Ohmori and Yokoyama [10] as one of the useful findings for detecting early esophageal neoplasms among multiple Lugol voiding areas in the inflamed esophagus. Shimizu et al. [3] reported that HGIN could be identified as iodineunstained areas that are more distinct and reddish than LGIN when the brown color of iodine solution in the normal region has faded after the staining. This is because there is almost no remaining glycogen-containing epithelium in HGIN. Recognition of PCS in the Lugol voiding area suggests the existence of carcinoma. With NBI, PCS is strongly emphasized and observed as a shiny silver color change, which we call 'metallic silver sign' or 'MSS'.

\section{IPCL Classification}

IPCL in the esophagus is usually observed as brown dots under NBI observation. IPCL appears in the plane closely above the muscularis mucosae branched from obliquely running vessels, and is observed as green vessels [11]. Inoue [5] categorized the surface microvasculature of the esophagus into five groups according to the irregularity of shape and degree of each IPCL's dilation. Type I represents normal or minimal change of IPCL. Type I and II usually lack a clear boundary, corresponding to benign pathology including inflammatory change and LGIN. Type III is defined as IPCL with minimal change of caliber, which usually has a clear boundary with background mucosa. Type III are thought to be borderline lesions including LGIN. In contrast, irregularly dilated IPCL with 
clear area formation is called IPCL type V, which corresponds to malignant pathology including HGIN with high probability. Type IV is the IPCL change between type III and V. Type IV indicates benign change such as LGIN with a probability of approximately $50 \%$. Therefore, the lesions that are categorized into type IV require consideration for removal, which enables further pathological evaluation. Type $\mathrm{V}$ is equivalent to definite malignant pathology including HGIN, which basically requires treatment including EMR and ESD. This classification simply offers information of whether the pathological confirmation should be performed by differentiating type III and IV. Recently, Kaga et al. [12] evaluated the detailed vascular structure pathologically and concluded that differences in vessels between IPCL type III and IV are highlighted by two factors: increased vessel caliber and prolongation of IPCLs toward the surface.

\section{Microvascular Patterns of Superficial Esophageal Cancers by Magnifying Endoscopy}

Arima et al. [7] classified the microvascular pattern into 4 categories according to the shape and irregularity of the surface microvessels. Type 1 is characterized by thin, linear capillaries in the subepithelial papilla and is generally seen in normal mucosa. Type 2 is characterized by distended, dilated vessels, and the shape of capillaries in the subepithelial papilla is preserved. Type 2 is generally seen in inflammatory lesions. Type 3 is characterized by spiral vessels with an irregular caliber and crushed vessels with red spots, and the arrangement of the vessels is irregular. Type 3 is generally seen in T1a-EP or T1a-LPM cancers. Type 4 has 4 subcategories including multiple layered, irregularly branched, reticular and avascular area (AVA). Type 4 is generally seen in cancers with T1a-MM or deeper invasion. AVAs and stretched type 4 vessels are seen in cancers with downward growth. AVAs were further subcategorized into 3 groups according to the size and it was reported that the size of AVAs was closely related to the depth of tumor invasion [7].

\section{Japanese Classification of Magnifying Endoscopy for Early Squamous Cell Carcinoma (Japan Esophageal Society) [8]}

However, the two major classifications mentioned above are considered to be somewhat complicated for global use. Therefore, the Japan Esophageal Society de- cided to create a new simplified classification. The new classification is composed of two major criteria. One is the shape and width of vessels, and the other is the size of the AVA. The vessels observed by magnified endoscopy were classified into two groups, types A and B.

Type A is vessels with mild or no atypia of IPCL. Type $B$ is IPCL with atypia including dilatation, meandering, caliber change and uneven form in each vessel. A lesion with type A strongly suggests intraepithelial neoplasia, whereas type B indicates SCC. Type B was subclassified into 3 groups, and the invasion depth of B1, B2 and B3 was consistent with T1aEP or LPM, T1aMM or T1bSM1 and T1bSM2, respectively. In addition, AVA was divided into three groups according to the tumor size. AVA small, AVA middle and AVA large were defined as $0.5 \mathrm{~mm}$ or less, between 0.5 and $3 \mathrm{~mm}$, and $3 \mathrm{~mm}$ or larger, respectively. The accuracy of diagnosis using this method was $90 \%$. They concluded that the new classification of magnified endoscopy is simple and useful for diagnosis of invasion depth of esophageal SCC.

\section{Background Coloration}

We have reported the importance of the color change in the epithelia between IPCLs (background coloration; $\mathrm{BC}$ ) in differentiating early SCC in the esophagus from benign lesions including inflammatory changes [13]. The $\mathrm{BC}$ has been additionally noted as intravascular $\mathrm{BC}$ in the Japanese classification of magnifying endoscopy for early SCC.

Ishihara et al. [14] reported that both the background color change and dilated IPCL was important in diagnosing esophageal SCC among any other early changes such as brownish dots (dilated IPCL), tortuous IPCL, elongated IPCL, caliber change in IPCL, variety in IPCL shapes, demarcation line and protrusion or depression. Also, Kanzaki et al. [15] statistically analyzed the cause of this color change. They reported that it may be related to thinning of the keratinous layer, caused by neoplastic cell proliferation and thinning of the epithelium. However, NBI mechanically reflects the wavelength that is specific to hemoglobin $(\mathrm{Hb})$. Therefore, we have come to realize that the color change might be related to the extravascular $\mathrm{Hb}$ component in the cancer area.

\section{Evaluation of $B C$}

After a brownish area in esophagus was found with $\mathrm{NBI}$, the lesions were observed with NBI magnification in order to evaluate the presence of $\mathrm{BC}$. A lesion was record- 
Fig. 1. a Iodine staining demonstrated a well-demarcated, slightly depressed lesion on the mid-esophagus. b Using NBI magnification, the color of the epithelia between each dilated IPCL in the lesion was altered to a brownish color compared to the surrounding whitish mucosa. This lesion was clearly positive for BC. c An $\mathrm{Hb}$ immunostaining image of the surrounding noncancerous area. Immunopositivity for anti-human $\mathrm{Hb}$ antibody was negative. d $\mathrm{An} \mathrm{Hb}$ immunostaining image of the cancer area. Immunopositivity was observed as intensely as $\mathrm{Hb}$ in the vessels.
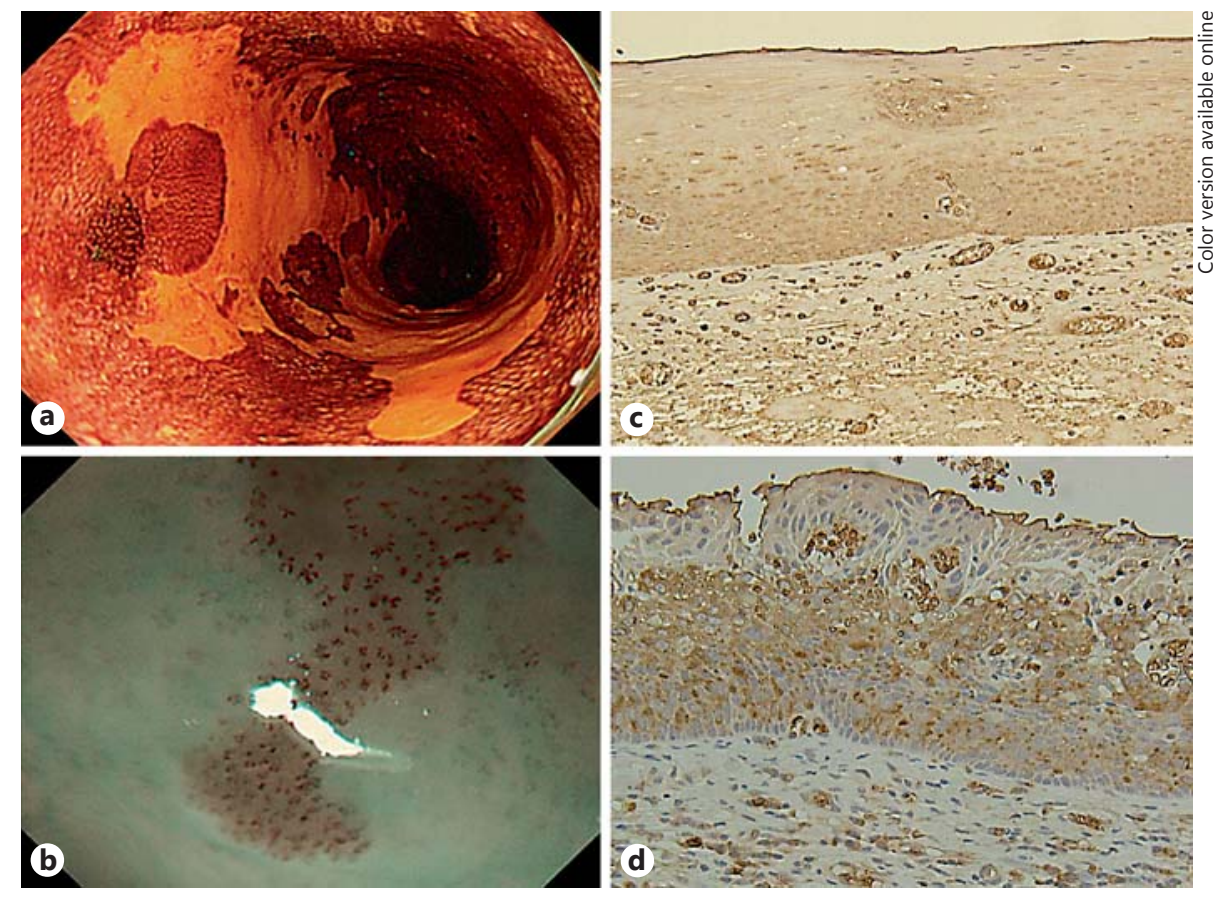

ed as $\mathrm{BC}$ positive when distinct color change in the area between IPCLs was seen. When there was no color change in this area, the lesion was regarded as $\mathrm{BC}$ negative.

In our current study including 223 lesions of early pharyngo-esophageal SCC, 194 lesions (86.6\%) were BC positive, only $3(2.5 \%)$ of which were pathologically diagnosed as benign (table 1). Sensitivity, specificity and overall accuracy of $\mathrm{BC}$ in differentiating malignancy from benign pathology were $91.1,71.4$ and $89.4 \%$, respectively.

\section{What Makes the Color Change?}

The cause of this phenomenon is still unclear. To begin with, NBI is a technique that enables us to exclusively identify the wavelength of $\mathrm{Hb}$. Therefore, we speculated the involvement of intra- or extravascular $\mathrm{Hb}$ in the cancer area. We applied an immunohistochemical approach using anti-human $\mathrm{Hb}$ antibody to evaluate the correlation between $\mathrm{BC}$ and the $\mathrm{Hb}$ component. Figure $1 \mathrm{c}$ and $\mathrm{d}$ show an immunopathological feature from both the $\mathrm{Hb}$ positive cancerous area and negative surrounding noncancerous area. Preliminary results revealed that there was a significant correlation between $\mathrm{Hb}$ immunopositivity and pathology. The rate of correlation between $\mathrm{BC}$ and $\mathrm{Hb}$ positivity was as high as $80.9 \%$. Interestingly, an immunofluorescent image showed that $\mathrm{Hb}$ positivity was mainly observed within cytosol of cancer cells outside of the nuclei with clear boundaries (fig. 2a, b).
Table 1. Correlation between BC positivity and pathology

\begin{tabular}{lrcr}
\hline & n & Inf./LGIN & HGIN/SCC \\
\hline $\mathrm{BC}(-)$ & 29 & $10(43.9 \%)$ & $19(56.1 \%)$ \\
$\mathrm{BC}(+)$ & 194 & $3(2.5 \%)$ & $191(97.5 \%)$ \\
\hline Total & 223 & 13 & 210 \\
\hline
\end{tabular}

The overall accuracy in diagnosing malignancies (HGIN/SCC) from benign change was $90.1 \%(10+191 / 223)$. Sensitivity was $91.0 \%(191 / 219)$, specificity was $77.0 \%$ (10/13), positive predictive value was $98.5 \%(191 / 194)$ and negative predictive value was $34.5 \%$ $(10 / 29)$.

Furthermore, real-time polymerase chain reaction, RT-PCR, was performed to quantify the $\mathrm{Hb}-\beta$ expression of both SCC and surrounding noncancerous mucosa. RT-PCR results revealed that the $\mathrm{Hb}-\beta$ mRNA expression rate was three times higher in the cancer area than the surrounding noncancerous area $(\mathrm{p}<0.05)$. In situ hybridization of $\mathrm{Hb}-\beta$ mRNA also confirmed the contribution of $\mathrm{Hb}-\beta$ expression in the cancer areas. These results support the belief that the $\mathrm{Hb}$ might be produced inside the cancer cells. 
Fig. 2. a Conventional HE staining revealed the T1a-EP esophageal SCC. b An immunofluorescent image (blue indicates nuclei; yellow-brown indicates anti-human $\mathrm{Hb}$ antibody). Intravascular RBCs are intensely positive (arrows). Similarly, SCC cells are positive in the cancer cells as well, especially in the supposed cytoplasm around the nuclei that are stained blue.
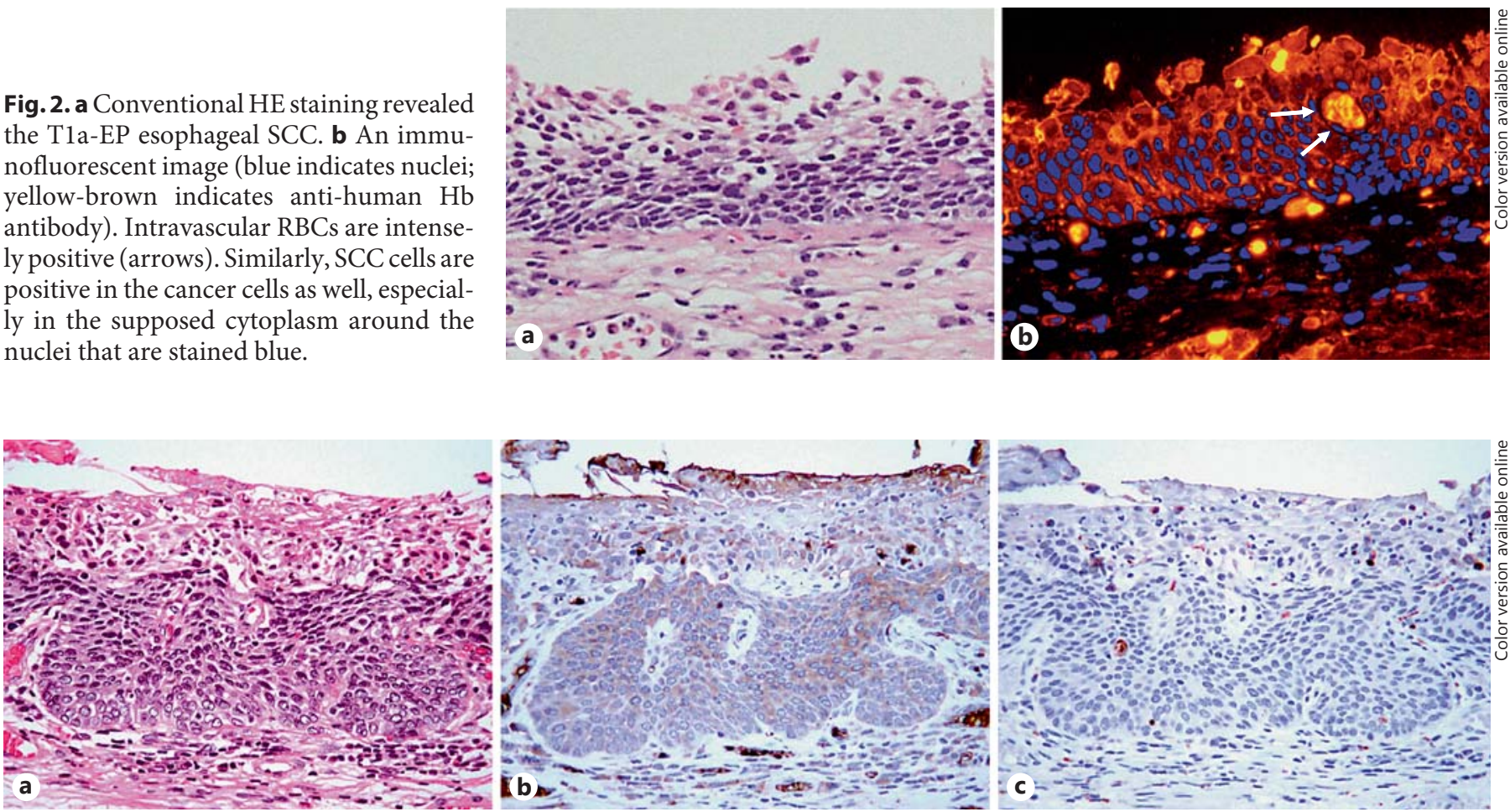

Fig. 3. An immunohistological image using anti-human CD68 antibody. Immunostaining showed strong positivity consistent with the cancer area, which was confirmed by conventional HE staining. On the other hand, immunopositivity for anti-human CD68 antibody was only slightly seen in the surface area of the lesion. a HE staining. b Anti-human $\mathrm{Hb} \mathrm{Ab}$ immunostaining. c Anti-human CD68 Ab immunostaining.

\section{Endocytoscopy}

Contact endoscopy was first described by Hamou [20] in 1979 as a method of microhysteroscopy to examine the surface of the genital tract at high magnification. Tada and Uozumi [21] first described the ultra-high magnifying endoscope in the gastrointestinal tract in 1982. Conventional magnification endoscopy enables detailed evaluation of gastrointestinal mucosa. Furthermore, endocytoscopy with ultra-high magnification allows in vivo observation of cellular atypia during routine endoscopic examination [22-24]. This novel technique opened the door to the possibility of 'optical biopsy', which enables us to obtain pathological diagnosis without taking biopsies. It is predicted that the incidence of cardiovascular or cerebrovascular complications would significantly increase. Optical biopsy using endocytoscopy will be of great help in the sense of saving time, money and perioperative risk due to interruption of anticoagulant therapy. The establishment of common diagnostic criteria of endocytoscopy imaging is essentially required. of BC.

10 


\section{Conclusion}

Recent advancement in the technology of image-enhanced endoscopy allows us to evaluate in detail the surface structure of the gastrointestinal tract. With the introduction of a newly constructed classification by the Japan Esophageal Society, diagnostics of esophageal SCC could be united and spread widely. Also, many studies with the purpose of illuminating the underlying mechanism and determining the significance of each finding have been conducted. It is important that we understand both the characteristics of the devices and the meaning of each finding comprehensively and precisely.

\section{Disclosure Statement}

The authors declare that they have no conflict of interests in connection with this paper.

\section{References}

- 1 Takubo K, Aida J, Sawabe M, Kurosumi M, Arima M, Fujishiro M, Arai T: Early squamous cell carcinoma of the oesophagus: the Japanese viewpoint. Histopathology 2007;51: 733-742.

-2 Muller JM, Erasmi H, Stelzner M, Zieren U, Pichlmaier H: Surgical therapy of oesophageal carcinoma. Br J Surg 1990;77:845-857.

-3 Shimizu Y, Omori T, Yokoyama A, Yoshida T, Hirota J, Ono Y, Yamamoto J, Kato M, Asaka M: Endoscopic diagnosis of early squamous neoplasia of the esophagus with iodine staining: high-grade intra-epithelial neoplasia turns pink within a few minutes. J Gastroenterol Hepatol 2008;23:546-550.

-4 Yokoyama A, Ohmori T, Muramatsu T, Yokoyama T, Okuyama K, Makuuchi H, Takahashi H, Higuchi S, Hayashida M, Maruyama $\mathrm{K}$, Ishii H: Short-term follow-up after endoscopic mucosectomy of early esophageal cancer and aldehyde dehydrogenase-2 genotype in Japanese alcoholics. Cancer Epidemiol Biomarkers Prev 1998;7:473-476.

5 Inoue $\mathrm{H}$ : Magnification endoscopy in the esophagus and stomach. Dig Endosc 2001; 13(suppl):40-41.

6 Kumagai Y, Inoue H, Nagai K, Kawano T, Iwai T: Magnifying endoscopy, stereoscopic microscopy, and the microvascular architecture of superficial esophageal carcinoma. Endoscopy 2002;34:369-375.

7 Arima M, Tada M, Arima H: Evaluation of microvascular patterns of esophageal cancers by magnifying endoscopy. Esophagus 2005;2: 191-197.

8 Oyama T, Ishihara R, Takeuchi M, Hirasawa $\mathrm{D}$, Arima $\mathrm{M}$, Inoue $\mathrm{H}$, Goda $\mathrm{K}$, Tomori $\mathrm{A}$, Monma K: Usefulness of Japan Esophageal Society Classification of Magnified Endoscopy for the Diagnosis of Superficial Esophageal Squamous Cell Carcinoma. Gastrointest Endosc 2012;75(suppl):AB456.
9 Mori M, Adachi Y, Matsushima T, Matsuda $\mathrm{H}$, Kuwano H, Sugimach K: Lugol staining pattern and histology of esophageal lesions. Am J Gastroenterol 1993;88:701-705.

10 Ohmori T, Yokoyama A: Clinical usefulness of pink-color sign (Japanese). Gastroenterol Endosc 2001;43(suppl 2):S1613.

11 Inoue H: Magnifying endoscopic diagnosis of tissue atypia and cancer invasion depth in the area of pharyngo-esophageal squamous epithelium by NBI enhanced magnification image: IPCL pattern classification; in Cohen J (ed): Advanced Digestive Endoscopy: Comprehensive Atlas of High Resolution Endoscopy and Narrowband Imaging. Malden, Blackwell, 2007, pp 49-66.

12 Kaga M, Inoue H, Kudo SE, Hamatani S: Microvascular architecture of early esophageal neoplasia. Oncol Rep 2011;26:1063-1067.

13 Minami H, Inoue H, Ikeda H, Satodate H, Hamatani S, Nakao K, Kudo SE: Usefulness of background coloration in detection of esophago-pharyngeal lesions using NBI magnification. Gastroenterol Res Pract 2012;2012: 529782.

14 Ishihara R, Inoue T, Uedo N, Yamamoto S, Kawada N, Tsujii Y, Kanzaki H, Hanafusa M, Hanaoka N, Takeuchi Y, Higashino K, Iishi H, Tatsuta M, Tomita Y, Ishiguro S: Significance of each narrow-band imaging finding in diagnosing squamous mucosal high-grade neoplasia of the esophagus. J Gastroenterol Hepatol 2010;25:1410-1415.

-15 Kanzaki H, Ishihara R, Ishiguro S, Nagai K, Matsui F, Yamashina T, Ohta T, Yamamoto S, Hanaoka N, Hanafusa M, Takeuchi Y, Higashino $\mathrm{K}$, Uedo N, Iishi $\mathrm{H}$, Tomita Y: Histological features responsible for brownish epithelium in squamous neoplasia of the esophagus by narrow band imaging. J Gastroenterol Hepatol 2013;28:274-278.

16 Roesch-Ely M, Nees M, Karsai S, Ruess A, Bogumil R, Warnken U, Schnolzer M, Dietz A, Plinkert PK, Hofele C, Bosch FX: Proteomic analysis reveals successive aberrations in protein expression from healthy mucosa to invasive head and neck cancer. Oncogene 2007;26:54-64.
$>17$ in carcinomas and cancer cell lines. FASEB J 2007;21:2931-2938.

18 Biagioli M, Pinto M, Cesselli D, Zaninello M, Lazarevic D, Roncaglia P, Simone R, Vlachouli C, Plessy C, Bertin N, Beltrami A, Kobayashi K, Gallo V, Santoro C, Ferrer I, Rivella S, Beltrami CA, Carninci P, Raviola E, Gustincich S: Unexpected expression of $\alpha$ - and $\beta$-globin in mesencephalic dopaminergic neurons and glial cells. Proc Natl Acad Sci USA 2009;106:15454-15459.

19 Nishi H, Inagi R, Kato H, Tanemoto M, Kojima I, Son D, Fujita T, Nangaku M: Hemoglobin is expressed by mesangial cells and reduces oxidant stress. J Am Soc Nephrol 2008; 19:1500-1508.

20 Hamou JE: Microendoscopy and contact endoscopy. Brevet Français 1979;79:04168.

21 Tada M, Uozumi Y: A new method for the ultra-magnifying observation of the colon mucosa. Kyoto Prefect University Med 1982; 91:349-354.

22 Inoue H, Sasajima K, Kaga M, Sugaya S, Sato Y, Wada Y, Inui M, Satodate H, Kudo SE Kimura S, Hamatani S, Shiokawa A: Endoscopic in vivo evaluation of tissue atypia in the esophagus using a newly designed integrated endocytoscope: a pilot trial. Endoscopy 2006; 38:891-895.

23 Minami $\mathrm{H}$, Inoue $\mathrm{H}$, Yokoyama A, Ikeda $\mathrm{H}$ Satodate H, Hamatani S, Haji A, Kudo S: Recent advancement of observing living cells in the esophagus using CM double staining: endocytoscopic atypia classification. Dis Esophagus 2012;25:235-241.

24 Kumagai Y, Kawada K, Yamazaki S, Iida M, Ochiai T, Momma K, Odajima H, Kawachi H, Nemoto T, Kawano T, Takubo K: Endocytoscopic observation of esophageal squamous cell carcinoma. Dig Endosc 2010;22:10-16.
Significance of BC in Endoscopic

Detection of Early Esophageal SCC 